\title{
Low dose of mycophenolate mofetil is enough in desensitized kidney transplantation using rituximab
}

Chung Hee Baek', Hyosang Kim', Hoon Yu', Eunhye Shin ${ }^{1}$, Hyungjin Cho ${ }^{1}$, Won Seok Yang ${ }^{1}$, Duck Jong $\mathrm{Han}^{2}$ and Su-Kil Park ${ }^{1 *}$

\begin{abstract}
Background: Rituximab is widely used in kidney transplantation. However, it is not clear whether the conventional doses of maintenance immunosuppressant in rituximab-treated kidney transplantation (KT) are appropriate. In our previous study, decreasing mycophenolate mofetil (MMF) dose due to infection did not increase the incidence of rejection or graft failure. Based on these experiences, we developed a new protocol with a lower dose of MMF and studied its clinical outcomes in rituximab-treated KT.

Methods: We enrolled all patients who underwent ABO-incompatible or human leukocyte antigen (HLA)-sensitized living donor $\mathrm{KT}$ with the new immunosuppressant protocol after preconditioning with rituximab, but without splenectomy from November 2011 to May 2013. Seventy-two patients (group 1) were consecutively enrolled in this study and followed until November 2013. Patients from our previous study served as control groups. Sixty-seven patients received KT using rituximab with a conventional dose of MMF (group 2), and 87 patients received ABO compatible KT without need for rituximab (group 3). Clinical outcomes, including rejection, infection, and graft survival, were compared between the groups. The $x^{2}$ test and Fisher's exact test were used for categorical variables, the Student's t-test and Mann-Whitney $U$ test were used for continuous variables, and a log-rank test was used for mortality analysis.
\end{abstract}

Results: Doses of postoperative MMF (g/day) were lower in group 1 than in the other groups $(1.03 \pm 0.19,1.48 \pm 0.34$ and $1.48 \pm 0.32 \mathrm{~g} /$ day at 1 week, $p<0.001)$. Infectious complications occurred more often in groups with conventional MMF doses (group 2 and 3) than in group 1 ( 16.7 vs. $37.3 \%, p=0.007$ and 16.7 vs. $34.5 \%, p=0.012$, respectively). Notably, group 1 showed a lower incidence of cytomegalovirus infection than group 2. However, reduction in MMF dose did not increase the incidence of acute rejection (4.2, 4.5 and $10.3 \%$ ). Only one graft failure occurred in group 2 due to vessel kinking after operation. There were no significant differences in the incidence of malignancy and mortality between groups.

Conclusions: A low MMF dose reduces infection without increasing rejection or graft loss and it may be appropriate to reduce the dose of MMF for rituximab-treated KT patients.

Keywords: Rituximab, Kidney transplantation, Immunosuppression

\footnotetext{
* Correspondence: skpark@amc.seoul.kr

${ }^{1}$ Division of Nephrology, Department of Internal Medicine, Asan Medical Center, University of Ulsan College of Medicine, Seoul, Republic of Korea Full list of author information is available at the end of the article
} 


\section{Background}

Rituximab, a chimeric anti-CD20 monoclonal antibody, is widely used in desensitization protocols these days. It was first used for $\mathrm{ABO}$ incompatible (ABOi) kidney transplantation (KT) combined with plasmapheresis and splenectomy in 2002 [1]. ABOi KT using rituximab instead of splenectomy as a desensitization protocol was reported within the following year [2]. Transplantations in HLA sensitized patients have recently been performed and $\mathrm{ABOi} / \mathrm{HLA}$-sensitized $\mathrm{KT}$ using rituximab has led to successful results [3-5].

Some retrospective studies have reported that rituximab is associated with an increased risk of infectious complications [6, 7]. However, a randomized, doubleblind, placebo-controlled study showed a trend toward fewer and milder rejections during the first 6 months in the rituximab group with no increase in infectious complications [5]. In our previous study [8], serious infectious complications occurred more often in the rituximab-treated KT. Therefore, the doses of mycophenolate mofetil (MMF) had to be decreased in the rituximab group to avoid or to cure serious infection. However, acute rejection was not observed with the reduced doses of MMF. After these experiences, we changed our immunosuppressant protocol for ABOi KT and HLA sensitized KT to a lower dose of MMF (1.0 g/day after postoperative week 1 in advance), and collected data prospectively without omitting any patients. So far, no guidelines have been suggested for a safe and effective maintenance immunosuppressive regimen in rituximab- and IL-2-receptor antibody-treated KT.

In this study, we evaluated our protocol of low dose of MMF in rituximab-treated $\mathrm{KT}$, focusing on its associations with reduced infectious complications without increased incidence of acute rejection.

\section{Methods \\ Patients}

We enrolled all patients who underwent ABOi or HLAsensitized, living-donor KT with the new immunosuppressant protocol after preconditioning with rituximab but without splenectomy (group 1) at the Asan Medical Center, a tertiary referral teaching hospital in Seoul, Korea, from November 2011 to May 2013. Seventy-two consecutive patients were enrolled for this study and followed until November 2013. No patients were lost to follow-up during the study period except in cases of death. Patients received tacrolimus, MMF, and corticosteroids as maintenance immunosuppressants according to the new protocol. Patients enrolled in our previous study [8] served as controls. Of these, 67 patients received desensitized KT using rituximab (group 2) and 87 patients received $\mathrm{ABO}$ compatible KT (group 3) between January 2009 and May 2011. They all received conventional doses of maintenance immunosuppressants. This study was approved by Asan Medical Center Institutional Review Board (2014-0724), and informed consent was obtained from all participants.

\section{Immunosuppression protocol}

The new immunosuppressant protocol for rituximabtreated renal transplantation is summarized in Fig. 1. Tacrolimus, MMF, and steroids were used in addition to rituximab and started 7-10 days before the operation. Tacrolimus was started at a dose of $0.075 \mathrm{mg} / \mathrm{kg}$ bid, and the target drug trough level was $10 \mathrm{ng} / \mathrm{mL}$ for postoperative weeks $1-2$ and $3-8 \mathrm{ng} / \mathrm{mL}$ thereafter. The starting dose of MMF was $500 \mathrm{mg}$ bid and elevated to $750 \mathrm{mg}$ bid from day 0 to day 7 postoperatively. After 1 week, the dose of MMF was reduced to $500 \mathrm{mg}$ bid. In groups 2 and 3, the conventional dose of $1.5 \mathrm{~g} /$ day of MMF was used for at least 1 month postoperatively. Rituximab was administered 7-10 days before the operation. We used $200 \mathrm{mg} /$ body rituximab for ABOi living donor transplantation, while $500 \mathrm{mg} /$ body rituximab was used in patients with a positive crossmatch on T-cell flow cytometry. Basiliximab, an anti-CD25 monoclonal antibody, was administered to all patients on the day of the operation and on postoperative day 4 . No patient received splenectomy or intravenous immunoglobulin (IVIG) injection. Several rounds of plasmapheresis were performed until the $\mathrm{ABO}$ isoagglutinin titer was reduced to less than 1:8 or T-cell flow cytometry $\left(\mathrm{COBE}^{\bullet}\right.$ Spectra, CaridianBCT, USA) was negative.

\section{Diagnosis of rejection}

Acute rejection was diagnosed on the basis of the Banff criteria, and C4d staining was performed in all specimens. Protocol biopsies were not routinely performed. Renal biopsies were performed to confirm rejection when clinically suspected by elevated serum creatinine.

\section{Definition of infection}

Cytomegalovirus (CMV) infection was defined as positivity on a CMV antigenemia assay $\geq 50$ cells/ 200,000 white blood cells and treated with ganciclovir. When plasma BK virus DNA was greater than 10,000 copies/ml, BK virus infection was diagnosed regardless of nephropathy. The CMV antigenemia assay and BK virus PCR were performed as previously reported, and the definition of infection was the same as the previous study [8]. CMV prophylaxis was not performed routinely because our nation-wide insurance system supports oral valganciclovir prophylaxis only for a case of donor's CMV IgG is positive and recipient's CMV IgG is negative. Instead, we checked CMV antigenemia routinely at $1,2,3,4,6,8,12,16,20$ and 24 weeks after KT and performed preemptive therapy 


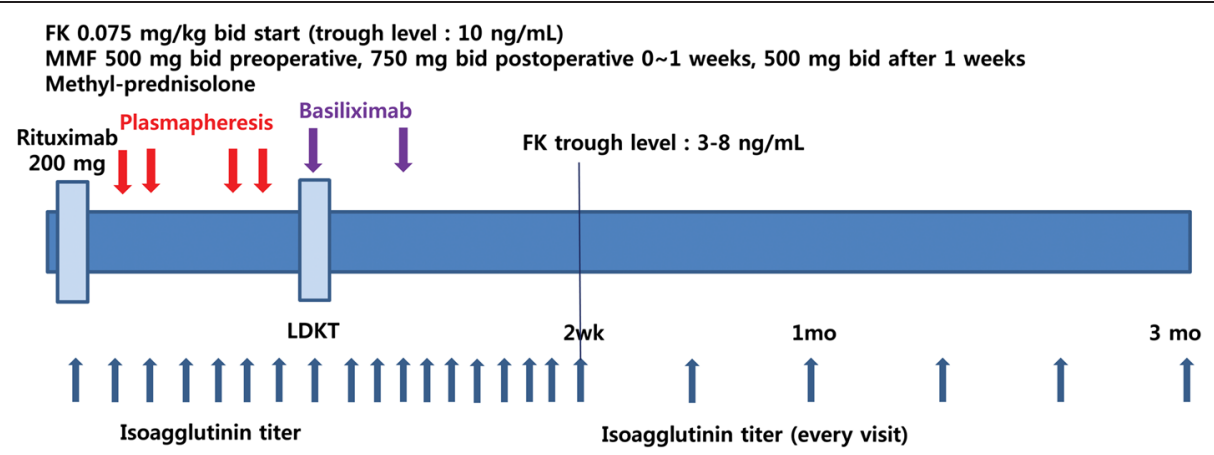

Fig. 1 New immuonosuppressive protocol. Tacrolimus, mycophenolate mofetil (MMF) and steroids are used in addition to rituximab and started 7-10 days before the operation. Tacrolimus is started at a dose of $0.075 \mathrm{mg} / \mathrm{kg}$ bid, and the target drug trough level is $10 \mathrm{ng} / \mathrm{mL}$ for the first 2 postoperative weeks and 3-8 ng/mL after 2 weeks. MMF is started at a dose of $500 \mathrm{mg}$ bid before the operation, and $750 \mathrm{mg}$ bid is used for the first postoperative week. After 1 week, MMF dose is reduced to $500 \mathrm{mg}$ bid

[9]. All patients received oral trimethoprim/sulfamethoxazole $(80 / 400 \mathrm{mg})$ for pneumocystis pneumonia prophylaxis until 6 months post-operation.

\section{Statistical analysis}

Statistical analyses were performed with SPSS 21 (IBM Corp., Armonk, NY, USA). Data are expressed as mean \pm standard deviation or counts and percentages. For categorical variables, the $\chi^{2}$ test and Fisher's exact test were used. Continuous variables were compared using the Student's $t$-test or Mann-Whitney $U$ test. Mortality rates were evaluated by the KaplanMeier method with a log-rank test. All statistical tests were two-tailed and $P$ value $<0.05$ was considered significant.

\section{Results}

\section{Baseline clinical characteristics}

Seventy-two patients were enrolled in group 1 (reduced dose of MMF), and 67 and 87 patients were enrolled in groups 2 and 3 (conventional dose of MMF), respectively. The mean age was not significantly different between groups 1 and 3, but group 2 patients were significantly older than group 1 patients $(40.92 \pm 10.20$ years vs. $44.88 \pm 11.65$ years, $p=0.034)$. In addition, the donors of group 1 were younger than the donors of group $3(39.63 \pm 10.95$ vs. $43.00 \pm$ 9.90 years, $p=0.043)$. There were more glomerulonephritis-caused cases of end stage renal disease in group 1. Most patients in all groups were positive for CMV IgG, and the CMV IgG serostatus of donor-recipient pairs was not different between groups. The mean follow-up time was $14.89 \pm 6.01$, $12.63 \pm 7.59$, and $14.05 \pm 8.17$ months in groups $1-3$, respectively, without significant difference. Baseline characteristics are summarized in Table 1.

\section{Levels and doses of immunosuppressant}

MMF doses were significantly lower in group 1 than in group 3 (Table 2). However, MMF doses in group 1 were significantly lower compared with group 2, predominantly in the early phase after transplantation, as MMF doses in group 2 had to be decreased due to infectious complications in many cases. Tacrolimus levels were not different between groups posttransplantation. The doses of methyl-prednisolone were lower in group 1 than in group 2. However, there was no difference between groups 1 and 3 .

\section{Incidence of infection}

The incidence of infection was lower in group 1 than in the other groups (Table 3). Although the incidence of each infection (CMV, BKV infection, urinary tract infection, pneumonia and sepsis) was not significantly different between groups 1 and 3, the total incidence was significantly lower in group 1 . In addition, group 1 showed significantly lower incidence of CMV infection compared with group $2(2.8$ vs. $16.4 \%, p=$ 0.007). Pneumonia and sepsis also showed trends of lower incidence in group 1 than in group 2 (1.4 vs. $9.0 \%, p=0.056$ and 0 vs. $6.0 \%, p=0.051$, respectively).

\section{Graft rejection and serum creatinine levels}

Acute cellular rejection occurred significantly more often in group 3 than in group 1 (10.3 vs. $1.4 \%, p=$ 0.023). There were no significant differences in other types of graft rejection between groups 1 and 3 (Table 4). Hyperacute rejection did not occur in any group. Chronic rejection occurred in a group 3 patient $(0.6 \%)$. There were 2 cases $(2.8 \%)$ of antibodymediated rejection in group 1 . However, there were no significant differences in rejection rates between groups 1 and 2. Serum creatinine levels are summarized in Table 5. Although there was no significant 
Table 1 Baseline clinical characteristics

\begin{tabular}{|c|c|c|c|c|c|}
\hline & \multirow{3}{*}{$\begin{array}{l}\text { Low dose MMF } \\
\text { Rituximab(+) } \\
\text { Group } 1(n=72)\end{array}$} & \multicolumn{2}{|c|}{ Conventional dose MMF } & \multirow[t]{3}{*}{$\mathrm{p} 1^{\mathrm{a}}$} & \multirow[t]{3}{*}{$\mathrm{p} 2^{\mathrm{b}}$} \\
\hline & & \multirow{2}{*}{$\begin{array}{l}\text { Rituximab(+) } \\
\text { Group } 2(n=67)\end{array}$} & \multirow{2}{*}{$\begin{array}{l}\text { Rituximab(-) } \\
\text { Group } 3(n=87)\end{array}$} & & \\
\hline & & & & & \\
\hline Sex (male/female) & $34 / 38$ & $42 / 25$ & $46 / 41$ & 0.088 & 0.526 \\
\hline Age (years) & $40.92 \pm 10.20$ & $44.88 \pm 11.65$ & $42.72 \pm 10.39$ & 0.034 & 0.273 \\
\hline Dialysis (hemo/peritoneal) & $50 / 13$ & $57 / 6$ & $54 / 13$ & 0.134 & 1.000 \\
\hline Dialysis duration (months) & $24.95 \pm 30.20$ & $32.11 \pm 33.47$ & $31.04 \pm 39.09$ & 0.210 & 0.324 \\
\hline Etiology of ESRD & & & & 0.043 & 0.006 \\
\hline Diabetes mellitus & 13 & 14 & 21 & & \\
\hline Hypertension & 11 & 12 & 23 & & \\
\hline Glomerulonephritis & 33 & 16 & 14 & & \\
\hline Vesicoureteral reflux & 0 & 4 & 1 & & \\
\hline Polycystic kidney disease & 3 & 5 & 5 & & \\
\hline Unknown etiology & 11 & 11 & 15 & & \\
\hline Other causes & 1 & 5 & 7 & & \\
\hline \multicolumn{6}{|l|}{ Past medical history } \\
\hline Diabetes mellitus & 14 & 15 & 23 & 0.682 & 0.348 \\
\hline Hypertension & 59 & 57 & 73 & 0.655 & 0.833 \\
\hline Hepatitis & 4 & 6 & 2 & 0.522 & 0.411 \\
\hline Autoimmune disease & 3 & 0 & 1 & 0.246 & 0.329 \\
\hline Malignancy history & 1 & 3 & 0 & 0.352 & 0.453 \\
\hline Mean follow up time (months) & $14.89 \pm 6.01$ & $12.63 \pm 7.59$ & $14.05 \pm 8.17$ & 0.053 & 0.455 \\
\hline ABO compatibility/T-flow & & & & 0.255 & \\
\hline ABO incompatible & 47 & 36 & - & & \\
\hline \multicolumn{6}{|l|}{ /T-flow(-) } \\
\hline ABO compatible & 14 & 21 & - & & \\
\hline \multicolumn{6}{|l|}{ /T-flow(+) } \\
\hline $\mathrm{ABO}$ incompatible & 11 & 10 & - & & \\
\hline \multicolumn{6}{|l|}{ /T-flow(+) } \\
\hline Donor to recipient & & & & 0.159 & \\
\hline Compatible & 14 & 21 & 87 & & \\
\hline$A \rightarrow B$ & 6 & 11 & - & & \\
\hline$B \rightarrow A$ & 9 & 8 & - & & \\
\hline $\mathrm{A} / \mathrm{B} \rightarrow \mathrm{O}$ & 24 & 18 & - & & \\
\hline$A B \rightarrow A / B / O$ & 19 & 9 & - & & \\
\hline HLA mismatch & $3 / 7 / 13 / 14 / 10 / 19 / 6$ & $4 / 0 / 7 / 20 / 7 / 18 / 11$ & $4 / 2 / 12 / 25 / 14 / 19 / 11$ & 0.065 & 0.335 \\
\hline \multicolumn{6}{|l|}{$(0 / 1 / 2 / 3 / 4 / 5 / 6)$} \\
\hline HLA class I mismatch & $5 / 14 / 25 / 16 / 12$ & $4 / 5 / 27 / 14 / 17$ & $5 / 8 / 34 / 23 / 17$ & 0.260 & 0.441 \\
\hline \multicolumn{6}{|l|}{$(0 / 1 / 2 / 3 / 4 /)$} \\
\hline HLA class II mismatch & $13 / 32 / 27$ & $6 / 33 / 28$ & $10 / 49 / 28$ & 0.296 & 0.275 \\
\hline \multicolumn{6}{|l|}{$(0 / 1 / 2)$} \\
\hline Donor sex (male/female) & $39 / 33$ & $34 / 33$ & $46 / 41$ & 0.735 & 0.875 \\
\hline Donor age (years) & $39.63 \pm 10.95$ & $41.88 \pm 11.65$ & $43.00 \pm 9.90$ & 0.241 & 0.043 \\
\hline
\end{tabular}


Table 1 Baseline clinical characteristics (Continued)

\begin{tabular}{|c|c|c|c|c|c|}
\hline \multicolumn{4}{|c|}{ Donor's relation with recipient } & \multirow[t]{6}{*}{0.822} & \multirow[t]{6}{*}{0.011} \\
\hline Parents & 8 & 7 & 8 & & \\
\hline Siblings & 17 & 20 & 36 & & \\
\hline Children & 14 & 11 & 6 & & \\
\hline Spouse & 27 & 21 & 22 & & \\
\hline Others & 6 & 8 & 15 & & \\
\hline \multicolumn{4}{|l|}{ CMV IgG serostatus } & \multirow[t]{4}{*}{0.178} & \multirow[t]{4}{*}{0.590} \\
\hline Donor+/Recipient+ & $70(97.2 \%)$ & $65(97.0 \%)$ & $86(98.9 \%)$ & & \\
\hline Donor+/Recipient- & 0 (0.0 \%) & $2(3.0 \%)$ & $0(0 \%)$ & & \\
\hline Donor-/Recipient+ & $2(2.8 \%)$ & $0(0.0 \%)$ & $1(1.1 \%)$ & & \\
\hline
\end{tabular}

Abbreviations: CMV cytomegalovirus, ESRD end stage renal disease, HLA human leukocyte antigen, MMF mycophenolate mofetil

a group 1 vs. group 2

broup 1 vs. group 3

Table 2 Doses of immunosuppressants

\begin{tabular}{|c|c|c|c|c|c|}
\hline & \multirow{2}{*}{$\begin{array}{l}\text { Low dose MMF } \\
\text { Rituximab(+) }\end{array}$} & \multicolumn{2}{|c|}{ Conventional dose MMF } & \multirow[t]{4}{*}{$\mathrm{p} 1^{\mathrm{a}}$} & \multirow[t]{4}{*}{$\mathrm{p} 2^{\mathrm{b}}$} \\
\hline & & Rituximab(+) & Rituximab(-) & & \\
\hline & Group 1 & Group 2 & Group 3 & & \\
\hline & $(n=72)$ & $(n=67)$ & $(n=87)$ & & \\
\hline \multicolumn{6}{|l|}{ Tacrolimus drug levels (ng/ml) } \\
\hline Pre KT $(72 / 67 / 87)$ & $6.99 \pm 2.57$ & $9.56 \pm 4.75$ & $11.11 \pm 6.58$ & $<0.001$ & $<0.001$ \\
\hline After 1 month (72/65/87) & $7.74 \pm 3.53$ & $9.17 \pm 3.22$ & $7.65 \pm 2.89$ & 0.015 & 0.847 \\
\hline After 3 months $(71 / 55 / 86)$ & $7.19 \pm 2.79$ & $7.64 \pm 2.54$ & $7.58 \pm 2.76$ & 0.349 & 0.380 \\
\hline After 6 months $(71 / 48 / 71)$ & $6.66 \pm 2.50$ & $7.11 \pm 2.11$ & $6.83 \pm 2.19$ & 0.304 & 0.664 \\
\hline After 1 year (49/39/48) & $5.77 \pm 1.81$ & $6.57 \pm 2.13$ & $5.77 \pm 2.16$ & 0.060 & 0.987 \\
\hline After 2 years $(6 / 4 / 15)$ & $7.18 \pm 1.28$ & $5.48 \pm 1.81$ & $5.51 \pm 3.16$ & 0.116 & 0.228 \\
\hline \multicolumn{6}{|c|}{ Mycophenolate mofetil doses ( $\mathrm{g} /$ day) } \\
\hline Pre KT $(72 / 67 / 87)$ & $1.44 \pm 0.15$ & $1.51 \pm 0.29$ & $1.55 \pm 0.17$ & 0.071 & $<0.001$ \\
\hline After 1 week (72/66/87) & $1.03 \pm 0.19$ & $1.48 \pm 0.34$ & $1.48 \pm 0.32$ & $<0.001$ & $<0.001$ \\
\hline After 2 weeks (72/66/87) & $1.00 \pm 0.15$ & $1.42 \pm 0.39$ & $1.48 \pm 0.31$ & $<0.001$ & $<0.001$ \\
\hline After 3 weeks $(72 / 66 / 87)$ & $1.00 \pm 0.17$ & $1.39 \pm 0.41$ & $1.46 \pm 0.32$ & $<0.001$ & $<0.001$ \\
\hline After 1 month (72/65/87) & $0.95 \pm 0.24$ & $1.26 \pm 0.42$ & $1.40 \pm 0.39$ & $<0.001$ & $<0.001$ \\
\hline After 3 months $(71 / 55 / 86)$ & $0.93 \pm 0.30$ & $1.14 \pm 0.51$ & $1.35 \pm 0.38$ & 0.007 & $<0.001$ \\
\hline After 6 months $(71 / 48 / 71)$ & $0.94 \pm 0.26$ & $1.07 \pm 0.50$ & $1.24 \pm 0.47$ & 0.095 & $<0.001$ \\
\hline After 1 year (49/39/47) & $0.93 \pm 0.28$ & $0.88 \pm 0.52$ & $1.23 \pm 0.41$ & 0.637 & $<0.001$ \\
\hline After 2 years $(6 / 4 / 16)$ & $1.08 \pm 0.20$ & $0.69 \pm 0.55$ & $1.28 \pm 0.45$ & 0.252 & 0.313 \\
\hline \multicolumn{6}{|c|}{ Methyl-prednisolone doses (mg/day) } \\
\hline Pre KT (72/67/87) & $16.00 \pm 0.00$ & $16.84 \pm 6.01$ & $15.91 \pm 0.86$ & 0.259 & 0.365 \\
\hline After 1 month $(72 / 65 / 87)$ & $11.11 \pm 1.68$ & $11.97 \pm 2.31$ & $11.59 \pm 2.70$ & 0.015 & 0.195 \\
\hline After 3 months $(71 / 55 / 86)$ & $8.03 \pm 1.49$ & $9.09 \pm 1.48$ & $8.16 \pm 2.06$ & $<0.001$ & 0.637 \\
\hline After 6 months $(71 / 48 / 71)$ & $6.25 \pm 1.82$ & $7.33 \pm 1.39$ & $6.87 \pm 3.59$ & 0.001 & 0.197 \\
\hline After 1 year (49/39/48) & $4.61 \pm 1.48$ & $5.33 \pm 1.80$ & $5.96 \pm 8.71$ & 0.042 & 0.289 \\
\hline After 2 years (6/4/16) & $3.00 \pm 2.45$ & $4.00 \pm 0.00$ & $3.75 \pm 1.44$ & 0.447 & 0.505 \\
\hline
\end{tabular}

Abbreviations: $K T$ kidney transplantation, MMF mycophenolate mofetil

a group 1 vs. group 2

broup 1 vs. group 3 
Table 3 Incidence of infection

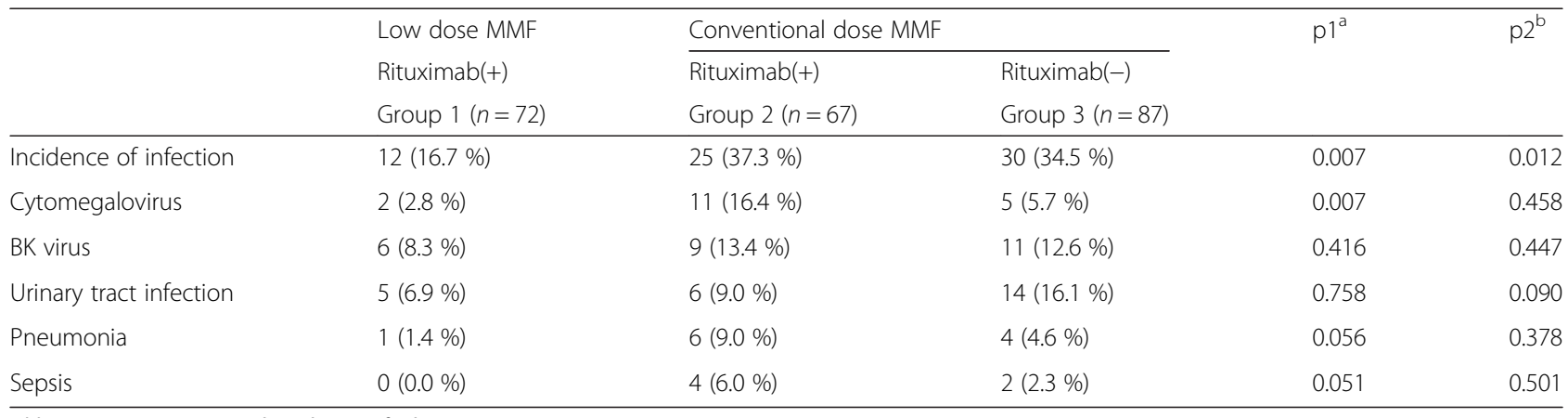

Abbreviations: MMF mycophenolate mofetil

aroup 1 vs. group 2

broup 1 vs. group 3

difference between groups 1 and 3, serum creatinine levels were lower in group 1 compared with group 2 .

\section{Incidence of malignancy and mortality}

Malignancy occurred in 2 patients $(3.0 \%)$ in group 2 and 1 patient $(1.1 \%)$ in group 3 , while there were no cases of malignancy in group 1 (Table 6). There was no significant difference in malignancy incidence between the groups. In group 2, malignancies were skin squamous carcinoma and parathyroid cancer. In group 3, one patient was diagnosed with colon cancer at 23 months post-operation. One patient $(1.4 \%)$ in group 1 died due to pneumonia aggravation, while there were 3 deaths (4.5\%) in group 2 (Fig. 2) caused by septic shock associated with urinary sepsis, uncontrollable fungal infective endocarditis and metabolic acidosis of unknown origin. One death (1.1\%) caused by pneumocystis pneumonia combined with bacterial infection was reported in group 3. Graft failure occurred in 1 patient in group 2 due to unexpected vessel kinking after the operation.

\section{Discussion}

Our present study findings suggest that lower doses of MMF can be safely used in rituximab-treated KT. There was no increase in rejection in patients who used low MMF doses, and infectious complications occurred less frequently.

Cyclosporine, azathioprine and corticosteroid used to be the maintenance immunosuppressants. However, most groups now use tacrolimus, MMF and corticosteroid. More intense immunosuppression has increased the concern about infections.

There have been various trials to reduce immunosuppressant levels. Typically, a dose of $375 \mathrm{mg} / \mathrm{m}^{2}$ or $500 \mathrm{mg} /$ body rituximab is used in $\mathrm{ABO}$ incompatible or HLA sensitized KT. However, good results have been reported with lower doses of rituximab in ABOi KT. Hiroki et al. [10] reduced the dose of rituximab from $500 \mathrm{mg} /$ body to $200 \mathrm{mg} /$ body and compared clinical outcomes and peripheral CD19 levels. Effective elimination of peripheral blood CD19 cells was observed until 24 months after treatment. In addition, there were no differences in creatinine levels, graft loss, or CMV infection between the groups. Another study compared the outcomes of $\mathrm{ABO}$ compatible and ABOi KT using low doses of rituximab $(100 \mathrm{mg} /$ $\mathrm{m}^{2}$ ) [11]. Estimated glomerular filtration rate, biopsyconfirmed rejection episodes, acute antibody-mediated rejection, and viral infection did not differ between the groups. The 5-year patient survival rate was $100 \%$ in both groups, while 5-year graft survival rates were $95 \%$ for $\mathrm{ABO}$ compatible and $100 \%$ for

Table 4 Graft rejection

\begin{tabular}{|c|c|c|c|c|c|}
\hline & \multirow{2}{*}{$\begin{array}{l}\text { Low dose MMF } \\
\text { Rituximab(+) }\end{array}$} & \multicolumn{2}{|c|}{ Conventional dose MMF } & \multirow[t]{3}{*}{$\mathrm{p} 1^{\mathrm{a}}$} & \multirow[t]{3}{*}{$\mathrm{p} 2^{\mathrm{b}}$} \\
\hline & & Rituximab(+) & Rituximab(-) & & \\
\hline & Group $1(n=72)$ & Group $2(n=67)$ & Group $3(n=87)$ & & \\
\hline Hyperacute rejection & $0(0 \%)$ & $0(0 \%)$ & $0(0 \%)$ & - & - \\
\hline Acute cellular rejection & $1(1.4 \%)$ & $3(4.5 \%)$ & $9(10.3 \%)$ & 0.352 & 0.023 \\
\hline Antibody-mediated rejection & $2(2.8 \%)$ & $0(0 \%)$ & $0(0 \%)$ & 0.497 & 0.203 \\
\hline Chronic rejection & $0(0 \%)$ & $0(0 \%)$ & $1(0.6 \%)$ & - & 1.000 \\
\hline
\end{tabular}

Abbreviations: MMF mycophenolate mofetil

a group 1 vs. group 2

broup 1 vs. group 3 
Table 5 Serum creatinine levels $(\mathrm{mg} / \mathrm{dL}$ )

\begin{tabular}{|c|c|c|c|c|c|}
\hline & \multirow{2}{*}{$\begin{array}{l}\text { Low dose MMF } \\
\text { Rituximab(+) }\end{array}$} & \multicolumn{2}{|c|}{ Conventional dose MMF } & \multirow[t]{3}{*}{$\mathrm{p} 1^{\mathrm{a}}$} & \multirow[t]{3}{*}{$\mathrm{p} 2^{\mathrm{b}}$} \\
\hline & & Rituximab(+) & Rituximab(-) & & \\
\hline & Group $1(n=72)$ & Group $2(n=67)$ & Group $3(n=87)$ & & \\
\hline Pre transplantation $(72 / 67 / 87)$ & $8.07 \pm 2.82$ & $8.51 \pm 2.91$ & $8.72 \pm 3.58$ & 0.371 & 0.213 \\
\hline After 1 month (72/66/87) & $1.03 \pm 0.60$ & $1.21 \pm 1.34$ & $1.03 \pm 0.33$ & 0.313 & 0.930 \\
\hline After 3 months (71/55/86) & $1.04 \pm 0.26$ & $1.17 \pm 0.29$ & $1.11 \pm 0.30$ & 0.012 & 0.153 \\
\hline After 6 months $(71 / 48 / 71)$ & $1.08 \pm 0.27$ & $1.21 \pm 0.31$ & $1.14 \pm 0.31$ & 0.012 & 0.181 \\
\hline After 1 year (49/39/48) & $1.02 \pm 0.26$ & $1.16 \pm 0.32$ & $1.07 \pm 0.29$ & 0.033 & 0.388 \\
\hline After 2 years (6/4/15) & $1.11 \pm 0.20$ & $0.98 \pm 0.21$ & $1.35 \pm 0.67$ & 0.338 & 0.397 \\
\hline
\end{tabular}

Abbreviations: MMF mycophenolate mofetil

${ }^{a}$ group 1 vs. group 2

bgroup 1 vs. group 3

ABOi transplants $(p=0.527)$. Some studies have even reported ABOi KT without using rituximab $[12,13]$.

There have been trials to minimize corticosteroid exposure. Kato et al. [14] reported results of an early steroid-withdrawal protocol in 130 patients using cyclosporine, MMF and methyl-prednisolone with basiliximab. The methyl-prednisolone was rapidly tapered and withdrawn on post-transplant day 14. The success rate of steroid withdrawal 12 months after transplantation in recipients of $\mathrm{ABOi}$ was $44 \%$. Galliford et al. [15] performed KT on 10 ABOi patients using 1 week of a steroids protocol with tacrolimus and MMF. Patient- and allograft-survival were $100 \%$ at 1-year post-transplantation in that study. Three patients experienced antibody-mediated rejection within 2 weeks of transplantation, although they were treated successfully. Oettl et al. [16] evaluated late steroid withdrawal after ABOi KT in 15 patients by performing protocol biopsies after 12 to 14 months. If the biopsy did not show signs of rejection, steroid was tapered and eventually stopped after 8 to 12 weeks. However, late steroid withdrawal was successfully performed in only 5 of 11 patients. The remaining 6 patients showed signs of mild acute rejection shortly after complete withdrawal or during steroid tapering.
Our center adopted a low dose of rituximab (200 mg/body) for ABOi KT at the end of 2009. The safety of the early steroid withdrawal protocol was left unproven, and it has been difficult to find data regarding the effectiveness of reduced maintenance immunosuppressant doses. However, in our previous study, we observed that reduced doses of MMF in rituximab-treated $\mathrm{KT}$ was safe [8]. CMV infection and pneumonia occurred more often in rituximab-treated KT. For management of infection, the dose of MMF was reduced, but it did not increase the incidence of acute rejection, and graft survival was $100 \%$. On the basis of this study, we changed our protocol. Our new protocol with lower dose of MMF showed good graft function and reduced incidence of infectious complications. It is a great burden to maintain longterm immunosuppressant treatment with resultant infectious risks in transplanted patients. Therefore, efforts to minimize immunosuppressant levels are justified and will be continued.

Our present study had some limitations. All of our study patients were Korean, and differences in physical and genetic factors between various ethnic groups might affect the clinical results of our protocol for rituximab- and IL-2 receptor antibody- treated KT patients. In addition, the follow-up period was short,

Table 6 Mortality and malignancy

\begin{tabular}{|c|c|c|c|c|c|}
\hline & \multirow{2}{*}{$\begin{array}{l}\text { Low dose MMF } \\
\text { Rituximab(+) }\end{array}$} & \multicolumn{2}{|c|}{ Conventional dose MMF } & \multirow[t]{3}{*}{$\mathrm{p} 1^{\mathrm{a}}$} & \multirow[t]{3}{*}{$p 2^{b}$} \\
\hline & & Rituximab(+) & Rituximab(-) & & \\
\hline & Group 1 $(n=72)$ & Group $2(n=67)$ & Group $3(n=87)$ & & \\
\hline Mortality & $1(1.4 \%)$ & $3(4.5 \%)$ & $1(1.1 \%)$ & 0.237 & 0.961 \\
\hline Graft failure & $0(0 \%)$ & $1(1.5 \%)$ & $0(0 \%)$ & 0.482 & - \\
\hline Malignancy & $0(0 \%)$ & $2(3.0 \%)$ & $1(1.1 \%)$ & 0.231 & 1.000 \\
\hline
\end{tabular}

Abbreviations: MMF mycophenolate mofetil

${ }^{\text {a }}$ group 1 vs. group 2

broup 1 vs. group 3 


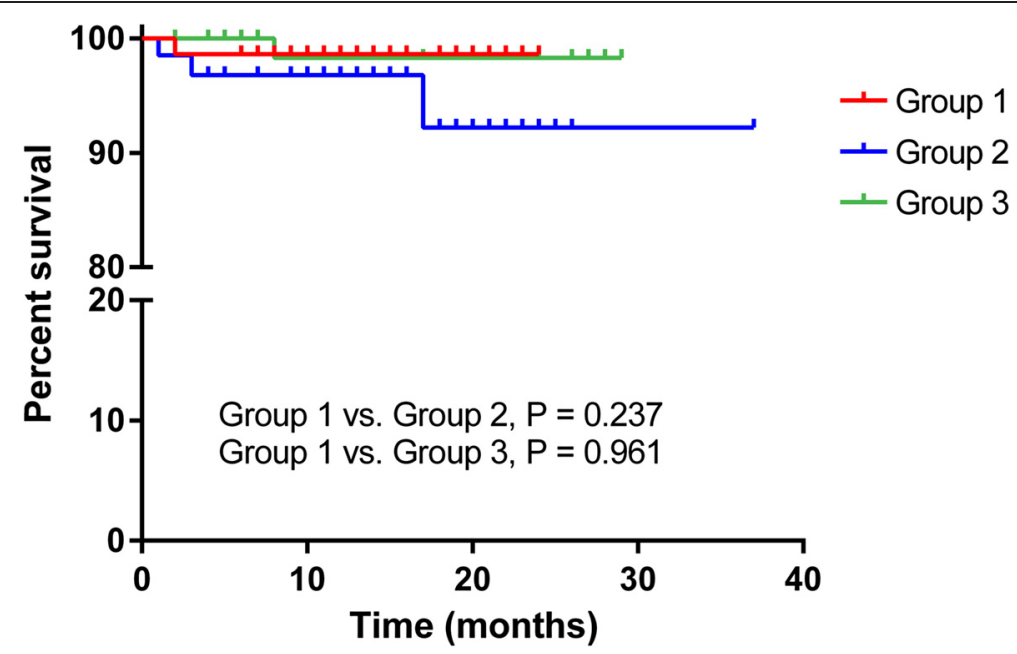

Fig. 2 Mortality. One patient in group 1 and one patient in group 3 died due to infectious complications. In addition, 3 patients died in group 2. The cause of death was uncontrolled infection in 2 of them

necessitating a longer-term follow-up study in the future for decisive clinical decision-making.

\section{Conclusions}

A protocol based on low doses of MMF decreases the infection rate without increasing the incidence of rejection or graft loss. This protocol might be useful for rituximaband IL-2 receptor antibody-treated KT, although a longterm clinical study is necessary to validate these findings.

\section{Abbreviations}

ABOi: ABO incompatible; CMV: Cytomegalovirus; HLA: Human leukocyte antigen; KT: Kidney transplantation; MMF: Mycophenolate mofetil.

\section{Competing interests}

The authors declare that they have no competing interests. The results presented in this paper have not been published previously in whole or part.

\section{Authors' contributions}

$\mathrm{CHB}$ analyzed the data and wrote the manuscript. HK analyzed the data and performed the study. HY, ES and HC collected the data. WSY and DJH performed the study. SKP performed the study, interpreted the data analyses and revised the manuscript. All authors participated in the study design and approved the final manuscript.

\section{Acknowledgements}

This study has no funding source.

\section{Author details}

'Division of Nephrology, Department of Internal Medicine, Asan Medical Center, University of Ulsan College of Medicine, Seoul, Republic of Korea. ${ }^{2}$ Department of Surgery, Asan Medical Center, University of Ulsan College of Medicine, Seoul, Republic of Korea.

Received: 18 September 2015 Accepted: 30 November 2015 Published online: 04 December 2015

\section{References}

1. Sawada T, Fuchinoue S, Teraoka S. Successful A1-to-O ABO-incompatible kidney transplantation after a preconditioning regimen consisting of antiCD20 monoclonal antibody infusions, splenectomy, and double-filtration plasmapheresis. Transplantation. 2002;74(9):1207-10. doi:10.1097/01.TP. 0000040990.15896 .76
2. Tyden G, Kumlien G, Fehrman I. Successful ABO-incompatible kidney transplantations without splenectomy using antigen-specific immunoadsorption and rituximab. Transplantation. 2003;76(4):730-1. doi:10.1097/01.TP.0000078622.43689.D4.

3. Fehr T, Stussi G. ABO-incompatible kidney transplantation. Curr Opin Organ Transplant. 2012;17(4):376-85. doi:10.1097/MOT.0b013e328355f013.

4. Kong JM, Ahn J, Park JB, Chung BH, Yang J, Kim JK, et al. ABO incompatible living donor kidney transplantation in Korea: Highly uniform protocols and good medium-term outcome. Clin Transplant. 2013;27(6):875-81. doi:10.1111/ctr.12249.

5. Tyden G, Genberg H, Tollemar J, Ekberg H, Persson NH, Tufveson G, et al. A randomized, doubleblind, placebo-controlled, study of single-dose rituximab as induction in renal transplantation. Transplantation. 2009;87(9): 1325-9. doi:10.1097/TP.0b013e3181a235fd

6. Habicht A, Broker V, Blume C, Lorenzen J, Schiffer M, Richter N, et al. Increase of infectious complications in ABO-incompatible kidney transplant recipients-a single centre experience. Nephrol Dial Transplant. 2011;26(12): 4124-31. doi:10.1093/ndt/gfr215.

7. Kamar N, Milioto O, Puissant-Lubrano B, Esposito L, Pierre MC, Mohamed $\mathrm{AO}$, et al. Incidence and predictive factors for infectious disease after rituximab therapy in kidney-transplant patients. Am J Transplant. 2010;10(1): 89-98. doi:10.1111/j.1600-6143.2009.02785.x

8. Baek CH, Yang WS, Park KS, Han DJ, Park JB, Park SK. Infectious risks and optimal strength of maintenance immunosuppressants in rituximab-treated kidney transplantation. Nephron extra. 2012;2(1):66-75. doi:10.1159/ 000337339.

9. Lee YM, Kim YH, Han DJ, Park SK, Park JS, Sung H, et al. Cytomegalovirus infection after acute rejection therapy in seropositive kidney transplant recipients. Transpl Infect Dis. 2014;16(3):397-402. doi: 10.1111/tid.12227.

10. Shirakawa H, Ishida H, Shimizu T, Omoto K, lida S, Toki D, et al. The low dose of rituximab in ABO-incompatible kidney transplantation without a splenectomy: A single-center experience. Clin Transplant. 2011;25(6):878-84. doi:10.1111/j.1399-0012.2010.01384.x.

11. Hatakeyama S, Fujita T, Murakami R, Suzuki Y, Sugiyama N, Yamamoto H, et al. Outcome comparison of ABO-incompatible kidney transplantation with low-dose rituximab and $\mathrm{ABO}$-compatible kidney transplantation: A singlecenter experience. Transplant Proc. 2014;46(2):445-8. doi:10.1016/j. transproceed.2013.09.036

12. Montgomery RA, Locke JE. ABO-incompatible transplantation: Less may be more. Transplantation. 2007;84(12 Suppl):S8-9. doi:10.1097/01.tp. 0000296032.12974.bb.

13. Barnett AN, Manook M, Nagendran M, Kenchayikoppad S, Vaughan R, Dorling $A$, et al. Tailored desensitization strategies in ABO blood group antibody incompatible renal transplantation. Transpl Int. 2014;27(2):187-96. doi:10.1111/tri.12234 
14. Kato Y, Tojimbara T, Iwadoh K, Koyama I, Nanmoku K, Kai K, et al. Early steroid withdrawal protocol with basiliximab, cyclosporine and mycophenolate mofetil in renal-transplant recipients. Int Immunopharmacol. 2006;6(13-14):1984-92. doi:10.1016/j.intimp.2006.07.018.

15. Galliford J, Charif R, Chan KK, Loucaidou M, Cairns T, Cook HT, et al. ABO incompatible living renal transplantation with a steroid sparing protocol. Transplantation. 2008;86(7):901-6. doi:10.1097/TP.0b013e3181880c0f.

16. Oettl T, Zuliani E, Gaspert A, Hopfer H, Dickenmann M, Fehr T. Late steroid withdrawal after $\mathrm{ABO}$ blood group-incompatible living donor kidney transplantation: High rate of mild cellular rejection. Transplantation. 2010; 89(6):702-6. doi:10.1097/TP.0b013e3181c9cc67.

Submit your next manuscript to BioMed Central and we will help you at every step:

- We accept pre-submission inquiries

- Our selector tool helps you to find the most relevant journal

- We provide round the clock customer support

- Convenient online submission

- Thorough peer review

- Inclusion in PubMed and all major indexing services

- Maximum visibility for your research 